Revista de Derecho

\title{
E-justicia en Ecuador: inclusión de las TIC en la administración de justicia
}

\section{E-Justice in Ecuador: Inclusion of ICTS in the Administration of Justice}

\author{
María Carolina Sacoto Romo \\ Abogada en Estudio Jurídico CMC Abogados \\ Cuenca, Ecuador \\ carolina.sacoto94@gmail.com \\ ORCID: 0000-0002-8799-8947

\section{Juan Manuel Cordero Moscoso} \\ Abogado en Estudio Jurídico CMC Abogados \\ Cuenca, Ecuador \\ jmcordero@cmc.com.ec \\ ORCID: 0000-0001-7080-1389
}

Artículo de investigación

DOI: https://doi.org/10.32719/26312484.2021.36.5

Fecha de recepción: 31 de diciembre de 2020

Fecha de revisión: 19 de enero de 2021

Fecha de aceptación: 16 de marzo de 2021

Fecha de publicación: 1 de julio de 2021

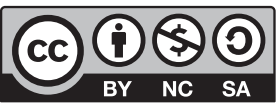




\section{RESUMEN}

Las nuevas tecnologías irrumpieron con fuerza y nos han situado en lo que se conoce como la 4.0 revolución industrial; han tenido incidencia directa en cada aspecto de nuestras vidas privadas así como en la administración pública, incluyendo a la justicia. Hoy en día, ya podemos hablar de un nuevo concepto que ha nacido de la introducción de las TIC al derecho: la e-justicia. En ese contexto, este artículo pretende en primer lugar, situar a la administración de justicia en el marco de lo que se considera la última revolución industrial, para luego intentar definir la e-justicia desde las ventajas que la implementación de esta representa; en un siguiente apartado, se abordará el aporte de las innovaciones y herramientas tecnológicas a la e-justicia en Ecuador, en base a cuatro usos troncales de las TIC relacionadas a la administración de justicia: la información, la gestión, la relación y la decisión, conforme la clasificación que hace Pere Fabra. El análisis se realizará desde una visión descriptiva y crítica, considerando la normativa vigente así como los adelantos existentes en otros países. La metodología de este trabajo es cualitativa, teórico exploratoria y se precisan como resultados los avances, así como los retos de la implementación de las TIC en la administración de justicia en Ecuador.

Palabras CLAVE: justicia electrónica, e-justicia, TIC, tecnología y derecho, digitalización de procesos, expediente electrónico, administración de justicia, justicia en Ecuador.

\section{ABSTRACT}

The irruption of new technologies has placed us in what is known as the 4.0 industrial revolution; they have had an impact on every aspect of our private lives as well as on public administration, including Justice. Today, there is already a new concept that was born from the introduction of ICTs to law: e-justice. In this context, this article aims, firstly, to place the administration of justice within the framework of what is considered the latest industrial revolution, then it will try to define e-justice taking into account the advantages that its implementation represents to approach the contribution of technological innovations to e-justice in Ecuador based on four core uses of ICTs related to the administration of justice: information, management, relationship and decision, according to the classification made by Pere Fabra. The analysis will be carried out from a descriptive and critical perspective, considering regulations in force as well as existing advances in other countries. The methodology of this work is qualitative, theoretical exploratory and are intented as results the progress as well as the challenges of the implementation of ICTs in the administration of justice in Ecuador.

KeYwords: electronic justice, e-justice, ICTs, legaltech, process digitalization, electronic filing, administration of justice, justice in Ecuador. 


\section{PLANTEAMIENTO GENERAL. ADMINISTRACIÓN DE JUSTICIA EN EL MARCO DE LA 4.0 REVOLUCIÓN INDUSTRIAL}

$\mathrm{O}$ las tecnológicas de gran magnitud se originaron hace varias décadas y se han desplazado a gran velocidad en todas las direcciones. Hemos hecho un cambio profundo, quizá sin darnos cuenta, en nuestra forma de vivir, y de relacionarnos con los demás, el mundo analógico fue desplazado por el mundo digital y hoy surfeamos en las olas de la 4.0 revolución industrial.

Esta metamorfosis tuvo un gran impulso en Ecuador con la pandemia por COVID-19, situación que impidió llevar adelante la administración de la justicia tal como se lo venía haciendo. En ese marco, Ecuador se encontró con multitud de desafíos siendo que en un Estado de Derecho se debe garantizar el principio de legalidad y oportunidad, preservando los derechos fundamentales de todas las personas y es así como se dieron los primeros pasos hacia la e-justicia; con ello, ha emergido gran inquietud acerca de los efectos de esta transformación digital y los inconvenientes que se puedan generar.

En este contexto, el presente artículo ofrece una visión descriptiva y crítica tanto de los modos en los que las TIC contribuyen a generar eso que se viene llamando justicia electrónica, así como de los avances y retos de la e-justicia en Ecuador.

\section{HACIA UNA NUEVA FORMA DE ENTENDER LA JUSTICIA: E-JUSTICIA}

La modernización de la administración de justicia ha introducido nuevas formas de llevar a cabo las actuaciones judiciales mediante el uso de las tecnologías de la información y la comunicación, avanzando a paso firme hacia la e-justicia. Este nuevo concepto supone el uso de una gran variedad de elementos tecnológicos en todos los procesos principales y conexos al impartir justicia.

Las definiciones que se dan de justicia electrónica son diversas, pero todas ellas reconocen que la penetrante transformación que existe tanto en la gestión de la información, en el manejo de expedientes judiciales, así como en los sistemas de comunicación relacionados al proceso y en la sustanciación de las causas, es una cuestión prioritaria y merecedora de atención y análisis.

Así, la también llamada justicia digital pretende poner la tecnología al servicio del derecho para lograr resultados que seríamos incapaces de conseguir sin usarla, su fin último es lograr una administración de justicia en la que la principal característica operativa sea la digitalización de los procesos desde su inicio hasta su conclusión, eli- 
minando barreras de tiempo y espacio; vemos que la implementación de estas herramientas representan innumerables ventajas que deberían ser aprovechadas por el Estado de modo que exista un empleo más eficiente de los limitados recursos disponibles.

Alcanzar este fin será sin duda alguna un avance significativo, pues conlleva un cambio sustancial en la forma de entender la justicia. Ese día se habrán superado numerosos retos y dificultades - mismos que se concretan más adelante- y estaremos listos para dar un paso más, nuevamente hacia lo desconocido, porque con toda seguridad, para ese entonces el mundo de la robótica y de la inteligencia artificial ya habrán desarrollado suficientes herramientas útiles para atender las nuevas necesidades de la sociedad.

Lograr una justicia $100 \%$ virtual toma tiempo, y requiere un plan de acción en el que se establezcan de forma clara los objetivos y los pasos para conseguirlos; de modo que, identificadas las necesidades, se han de comprender cuáles son las ventajas de realizar estos cambios para usarlos en pro de reinventar la forma de concebir la justicia.

Por mencionar algunos aspectos positivos, con la introducción de las TIC en la rama del derecho se podría apostar por la economía procesal, la celeridad, mayor seguridad en la valoración de las pruebas - siempre que sea posible - y mejor acceso a la justicia para la población en general, de escasos recursos y personas con movilidad limitada. También se podrá advertir, aunque en una fase avanzada, reducción de costos para el Estado ${ }^{1}$ y para las partes, acceso $24 / 7$ a la justicia, desde cualquier parte del mundo, e incluso apertura a los litigios trasfronterizos. Por otro lado, los profesionales de la justicia podrán ahorrar tiempo y optimizar su trabajo, tendrán alcance inmediato a la información y se evidenciará mayor confianza en la administración de justicia.

Estas enunciaciones ventajosas son aún más importantes en la medida en la que permitirán una disminución de las tareas repetitivas que realizan los especialistas del derecho; así, jueces y otros auxiliares de la justicia podrán dedicarse a la tarea para la cual fueron asignados a ese puesto: estudiar los casos, tomar decisiones y poner fin a las controversias que conozcan. En definitiva, las TIC abren puertas que permitirán cumplir, en mayor medida, las garantías, los derechos fundamentales y los principios procesales establecidos en la norma.

1. Estonia, por ejemplo, opera con uno de los presupuestos per cápita más bajos de toda la Unión Europea. Estonia e-estonia, "e-justicia”, Enterprise Estonia, accedido 26 de febrero, https://bit.ly/3uJvKNw. 


\section{HACIENDO EL CAMBIO: USOS DE LAS TIC EN LA ADMINISTRACIÓN DE JUSTICIA Y SUS AVANCES EN ECUADOR}

No se puede negar que una administración de justicia analógica, con juzgados congestionados y tiempos de espera insostenibles, han limitado, en gran medida, en Ecuador el acceso en igual de condiciones a la justicia, poniendo en grave peligro la tutela judicial efectiva del derecho consagrado en la Constitución de la República del Ecuador, ${ }^{2}$ más aún considerando que la Carta Magna, con total claridad, dispone que el Estado será el responsable por la inadecuada administración de justicia. ${ }^{3}$

Con estos antecedentes, el Consejo de la Judicatura para el año 2020 redobló esfuerzos en la modernización de la justicia mediante la implementación de herramientas digitales que permiten a profesionales del derecho en libre ejercicio profesional, a jueces, fiscales, instituciones públicas y privadas, e inclusive al público en general, realizar trámites judiciales en línea y acceder a información; considerando como objetivos principales promover la transparencia, celeridad y eficiencia en la tramitación de las causas judiciales. La modernización a la que nos hemos referido ha venido a Ecuador con la implementación del e-satje $2020 .^{4}$

Para precisar los usos de las TIC en la administración de justicia, y los avances que ha tenido Ecuador, hemos de tomar como referencia la clasificación que hace Pere Fabra, considerando dos criterios: el grado de complejidad técnica de la aplicación y el nivel de interacción que cada aplicación permite entre los diferentes operadores jurídicos. Siguiendo estas variables, según el autor, se determinan cuatro usos troncales de las TIC en la administración de justicia, siendo estos: la información, la gestión, la relación y la decisión.

\section{Tratamiento de LA INFORMACión}

Son varios los inconvenientes que surgen cuando existe un manejo físico de la documentación, por mencionar algunos: el deterioro que pueden sufrir los papeles por el paso del tiempo y la manipulación, el espacio físico que ocupan y la limitación de que solo una persona a la vez puede acceder al expediente, además de que la dupli-

2. Ecuador, Constitución de la República del Ecuador, Registro Oficial 449, 20 de octubre de 2008, art. 75.

3. Ibíd., art. 11.9.

4. Ecuador Función Judicial, “e-satje 2020”, Función Judicial, accedido 15 de noviembre, https://bit. ly/34Uad9J. 
cación de los documentos en físico necesaria para la tramitación de causas es costosa y contaminante. Estos y otros problemas se minimizan con el tratamiento digital de toda documentación.

En ese marco, se debe enfatizar en que el tratamiento de la información es uno de los usos más comunes al momento de emplear la tecnología. En su aplicación a la justicia, este uso específico también ha sido referido por otros autores como back office, ${ }^{5}$ contemplado como un eje vital de apoyo al desenvolvimiento de las actuaciones de justicia. Así, el procesamiento de textos y bases de datos es el primer paso que ha de darse para la consecución eficiente de los demás usos posibles, tal como se explicará en los apartados siguientes.

El tratamiento de información en Ecuador se ha llevado a cabo por medio de la organización en bases de datos tanto de los procesos judiciales signados con números y códigos de dependencia, así como de los profesionales del derecho que patrocinan cada causa, estas bases de datos se nutren de toda la información que los operadores de justicia y las partes ingresan a los sistemas.

A partir de estos datos relacionados y estructurados, los funcionarios son capaces de gestionar con mayor eficiencia los procesos a su cargo. Así, el tratamiento adecuado de la información permite realizar intercambios entre los juzgados y abogados, y por tanto, entre abogados y ciudadanos, enviar y recibir información de entidades públicas y otros auxiliares de la justicia en instantes. Por otro lado, el procesamiento de textos es eficiente y permite emitir providencias, autos y sentencias en base a plantillas de casos similares y relacionados que agilizan la gestión siempre que estos procesos no exijan un análisis particular del caso en concreto. ${ }^{6}$

Dentro de este punto también se deben considerar las bases de datos que se nutren de las últimas actualizaciones de normas, doctrina y jurisprudencia. En este ámbito, Ecuador cuenta con el sistema E-SIPJUR (Sistema de procesamiento de Jurispruden$\mathrm{cia}^{7}$ ) que permite ubicar precedentes jurisprudenciales obligatorios, resoluciones con fuerza de ley y sentencias, minimizando la posibilidad de impartir justicia con errores. En lo que se refiere a sistemas unificados electrónicos que contienen las últimas actualizaciones y reformas de normas o jurisprudencia, han tomado papel protagónico algunas empresas privadas.

5. Dory Reling, "E-justice: experiences with court IT in Europe", Buenas prácticas para la implementación de soluciones tecnológicas en la administración de justicia 1 (2011): 79-115, https://bit.ly/2Juu1ZJ.

6. Al respecto, en Estonia se trabaja en determinar qué tareas son las más lentas y repetitivas, de modo que puedan ser reemplazadas con sistemas automatizados con inteligencia artificial. Estonia e-estonia, "e-justicia".

7. Ecuador Función Judicial, "Sistema de Jurisprudencia", Función Judicial, accedido 20 de diciembre, https://bit.ly/3qOoYmv. 
A pesar de los constantes esfuerzos, hay que admitir que al momento en el país no se ha alcanzado el nivel de interoperabilidad deseado entre bases de datos que permitan cruzarlas con otras disponibles y verificar información para mitigar riesgos y evitar errores, ${ }^{8}$ pero se trabaja en ello y se espera alcanzar este objetivo a mediano plazo. Tampoco existe cabida aún para otros usos posibles relacionados a sistemas con técnicas de inteligencia artificial ya activos en otros países a los que nos referimos brevemente a continuación.

Países más desarrollados ya alcanzaron una interconexión eficiente de información, y por tanto, ya dejaron entrar al juego a la inteligencia artificial, estas naciones dedicadas a la investigación de las nuevas tecnologías aplicadas al derecho ya han perfeccionado y uniformado el lenguaje jurídico para obtener de esta manera mejores resultados al momento de realizar investigaciones en los buscadores. Otro avance significativo fue el primer sistema de enseñanza basado en casos, desarrollado por Kevin D. Ashley, profesor de la Universidad de Pittsburg, experto en modelado informático del razonamiento jurídico y cuestiones legales, sistema denominado CATO. ${ }^{9}$ Así también, la información legal en bases de datos se utiliza en sistemas de asesoría jurídica (Legal Advisory Systems), y en otras aplicaciones legales, Split-up o SHYSTER en Australia, ASHSD-II en Inglaterra, CHIRON en Estados Unidos, o LASTC en Tailandia son algunos ejemplos, siendo sistemas de expertos legales que basados en datos, utilizan inteligencia artificial para emular las habilidades de toma de decisiones de un experto humano en el campo del derecho específico del que se trate.

\section{EXPEDIENTE JUDICIAL ELECTRÓNICO ${ }^{10}$}

Convencionalmente, el expediente incluye una serie de documentos sobre las actuaciones judiciales distribuidos en varios cuerpos que se almacenan físicamente y que deben moverse de un lado al otro de modo que jueces y partes intervinientes puedan acceder a este. Con ánimo de alcanzar los objetivos de la e-justicia, y la eficiencia en el manejo de los casos, se pensó en extender el uso de las TIC y llevar la gestión de causas mediante un expediente judicial electrónico, entendiendo por este "al conjunto de datos, documentos, trámites y actuaciones electrónicas, así como de grabaciones

8. Estonia lo realiza mediante el sistema e-File. Estonia e-estonia, "e-justicia".

9. Kevin Ashley, "Designing Electronic Casebooks that Talk Back: The CATO Program", Jurimetrics, vol. 40, n. 3 (primavera de 2000): 275-319.

10. Ecuador, Código Orgánico General de Procesos, Registro Oficial 506, 22 de mayo de 2015, art. 115, 117, 202. Regula lo referente a la digitalización de documentos y documentos digitales. 
audiovisuales correspondientes a un procedimiento judicial, cualquiera que sea el tipo de información que contenga y el formato en el que se hayan generado". ${ }^{11}$

El e-satje en Ecuador es el primer avance hacia la gestión del expediente judicial electrónico, ${ }^{12}$ este implementa la Oficina Judicial Electrónica, la cual permite que el abogado patrocinador revise el casillero judicial electrónico en donde puede visualizar los anexos de los escritos presentados y de las providencias que se despachen, el estado de tramitación del proceso y la documentación que se ha incorporado, pero aún con ciertas limitaciones, como por ejemplo, el hecho de que sea un solo abogado de la defensa técnica el que acceda al sistema; igualmente, ya se dio inicio a la migración ordenada del expediente físico al expediente digital, con una mirada firme hacia el día en el que se tengan en Ecuador juzgados sin papel. En definitiva, este servicio pretende eliminar las barreras de tiempo y espacio y mejorar la seguridad sobre la documentación; sin lugar a duda, superada la necesidad de tener los documentos físicamente, se hace más sencilla la transmisión de información.

Ahora bien, para los clientes internos de la Función Judicial un sistema óptimo incluiría además otras competencias que permitan la gestión de los procesos y sirvan de apoyo a los funcionarios para la planificación y seguimiento de los casos que conocen, ${ }^{13}$ estas herramientas tecnológicas transversales son útiles para digitalizar actividades relacionadas a los procesos judiciales, permiten una cohesión eficiente de los diferentes profesionales de la justicia y mejoran su desenvolvimiento, ejemplos son la implementación de intranets o plataformas para la capacitación de operadores jurídicos.

Finalmente, sobre esta categoría hay que recalcar que también es de gran utilidad para el Estado al momento de levantar estadísticas en relación con los casos iniciados, en proceso y concluidos; estos sistemas correctamente manejados permitirán obtener ratios para evaluación de resultados, generación de KPI's ${ }^{14}$ que permitan medir la gestión realizada, análisis sociológicos, etc.

Conscientes de estos beneficios, Ecuador puso en marcha el denominado "Trámite web" ${ }^{15}$ dentro del e-satje 2020, una herramienta de gestión interna que permite a los integrantes del equipo jurisdiccional realizar el seguimiento de los procesos que les han sido asignados; se pretende como resultado un trabajo cooperado para facilitar el

11. España, Ley 18/2011 Reguladora del uso de las Tecnologías de la Información y la Comunicación en la Administración de Justicia, Boletín Oficial 160, 5 de julio de 2011.

12. Ecuador, Código Orgánico General de Procesos, art. 119 que permite el registro electrónico de actos procesales.

13. En Estonia existe el Sistema de información judicial (KIS) Estonia e-estonia, "e-justicia".

14. Por sus siglas en inglés Key Performance Indicator o Indicadores Clave de Desempeño.

15. Ecuador, Código Orgánico de la Función Judicial, en concordancia con el art. 147. 
flujo de la información entre operadores internos y externos, la gestión unificada de tareas y la visualización de documentación común para que cada individuo del sistema pueda conectar con los demás. Mientras ello ocurre, también se condensa información relevante para obtener índices sobre los resultados de modo que es más fácil identificar las falencias para superarlas como parte de un proceso de mejora continua.

En cuanto a las plataformas para impartir talleres, cursos, tutoriales y demás eventos similares, no se cuenta con un desarrollo propio para este fin, pero ello no ha impedido la labor constante que realiza la Función Judicial por medio de su órgano administrativo para capacitar a los usuarios de la justicia y dar acompañamiento a todos los interesados en este proceso de inclusión de las TIC; estas actividades se llevan a cabo mediante plataformas que están en el mercado.

En otros países ya se trabaja activamente para la implementación de la inteligencia artificial mediante el machine learning o procesamiento del lenguaje, se tienen asistentes virtuales como Alexa, que absuelven con facilidad consultas de los usuarios en un lenguaje sencillo, también se consideran chatbots web entrenados e incluso ya se ha apostado por que los sistemas informáticos aprendan de los datos con los que se alimentan y empiecen a actuar sin guía del ser humano en tareas recurrentes como la gestión automatizada de documentos para incluirlos en el expediente judicial electrónico y tareas básicas en otros procedimientos repetitivos que inicialmente las realizaba una persona.

Se mentiría si se dijera que Ecuador está en posibilidades de implementar herramientas como las mencionadas, la iniciativa del expediente judicial electrónico en Ecuador es reciente, ni siquiera está disponible aún en todo el territorio nacional porque su implementación se hará por fases, priorizando las unidades judiciales de primer nivel y cortes provinciales, en espera de que en el 2021 esté operando a nivel nacional. Con lo dicho, es evidente que estos procesos son nuevos y todavía no cerrados, pero con su iniciación ya se reconoce el esfuerzo que el órgano de justicia ha puesto en llegar a una virtualización integral de los procesos judiciales, ya se aprecia de a poco la desmaterialización de algunos procedimientos, y como consecuencia lógica, la reestructuración de infraestructuras técnicas y organizativas a nivel nacional para adaptarse al uso de las nuevas herramientas tecnológicas.

\section{RELACIÓN ENTRE OPERADORES JURÍDICOS}

Quienes aplican y vigilan el cumplimiento de las normas han de estar comunicados mediante medios eficaces, si la Función Judicial y órganos auxiliares logran una interacción adecuada con los profesionales del derecho que defienden los intereses de los ciudadanos, habrán logrado un contacto satisfactorio con los clientes finales. 
En este apartado se evalúan varios usos posibles, la descarga de formularios para ser llenados por los usuarios es uno de ellos. En Ecuador se pone a disposición de la ciudadanía formularios para la demanda, rebaja y aumento de pensiones alimenticias, mismas que no requieren patrocinio legal, pero en este caso deberán presentarse por la ventanilla física ya que solo los profesionales del derecho están facultados para ingresar peticiones por la ventanilla virtual; existe también un formulario para la presentación de demanda en proceso monitorio, aunque en este último sí se requiere obligatoriamente un defensor técnico. A este respecto, países pioneros en técnicas más avanzadas han implementado programas interactivos que llenan automáticamente los formularios, ya sea para requerir o para dar contestación a una variedad de asuntos legales tal como lo hizo en su momento el sistema EZLegalFile ${ }^{16}$ en California o la plataforma TurboCourt ${ }^{17}$ misma que en primer lugar permite la selección de la jurisdicción para luego proceder al llenado de la documentación conforme la norma vigente en el Estado escogido.

Otros usos de las TIC que se consideran en este apartado son la gestión de certificados electrónicos y la presentación de escritos y demandas en línea con firma electrónica. ${ }^{18}$ Ecuador los implementó con la Oficina Judicial Electrónica, mediante la cual se permite el ingreso de nuevas peticiones con sus respectivos anexos, también se permite el ingreso de escritos de procesos que actualmente ya se encuentran en trámite, y lógicamente de los procesos que sean ingresados virtualmente.

Un escalón superior y bastante controvertido es el de las actuaciones mediante videoconferencia en las diferentes etapas de un proceso judicial. Aunque en una implementación ideal de la e-justicia este es un aspecto primordial, lo cierto es que en este punto sí pueden verse comprometidos derechos y garantías fundamentales de la persona, al igual que principios procesales. El uso de estos medios telemáticos podría ser menos conflictivo si se utilizan en procesos de materia civil, tributaria o laboral, y sobre todo en audiencias en las que no tenga que producirse prueba compleja, audiencias preliminares o lecturas de sentencia, e inclusive para mediación o arbitraje. ${ }^{19}$

En Ecuador ya se reconoció la utilidad de estos medios audiovisuales, se ha pensado en personas con discapacidad, adultos mayores, controversias entre personas de distintos países, etc. Se sabe del potencial considerable que poseen estos medios para

16. Actualmente el sistema EZLegalFile ya no está disponible porque fue incorporado directamente a la página web oficial del Consejo Judicial de Santa Clara de California.

17. Turbo Court, "Prepare your court case online", accedido 17 de noviembre de 2020, https://bit.ly/3rq6NoO.

18. Definición de firma electrónica en Ecuador, Ley de Comercio Electrónico, Firmas Electrónicas y Mensajes de Datos, Registro Oficial 557, Suplemento, 17 de abril de 2002, art. 13.

19. En Ecuador Consejo de la Judicatura, Resolución n.$^{\circ}$ 039-2020, Registro Oficial 217, 4 de junio de 2020. 
promover el acceso transfronterizo a la justicia facilitando este tipo de litigios pues indudablemente reduce la complejidad y los costos.

Ya se había previsto el uso de videoconferencias para determinados casos tanto en el Código Orgánico General de Procesos (COGEP), ${ }^{20}$ así como en el Código Orgánico Integral Penal. ${ }^{21}$ Actualmente, la decisión de llevar a cabo una audiencia de forma presencial o de manera virtual es de cada uno de los juzgadores que conozcan la causa, previa valoración del caso concreto y ponderación de los derechos y principios jurídicos relacionados, para ello deben analizar las circunstancias específicas, el tipo de audiencia y convocarla para que se lleve a cabo de forma virtual siempre que se garanticen los principios que sustentan el sistema procesal.

$\mathrm{Al}$ respecto, la Corte Nacional de Justicia, ha emitido un protocolo para la realización de audiencias virtuales, señalando que estos sistemas han de pretender una administración de justicia ágil, efectiva y de calidad, reconociendo a su vez que es una medida necesaria para velar por la tutela judicial efectiva de los derechos y el debido proceso.

Así, el modus operandi del aparato de justicia va cambiando, hoy se trata de generar, como nunca antes, una sinergia entre autoridades y funcionarios judiciales, y las videoconferencias se muestran muy útiles para este fin. En ese sentido, las audiencias que se lleven a cabo usando medios telemáticos están sujetas a cumplir con las formalidades necesarias que garanticen la validez legal de estas, para responder a la publicidad de las audiencias, el interesado debe pedir autorización al juez para estar presente en la audiencia virtual, las audiencias serán grabadas en audio por el secretario para luego incorporarlo al expediente. Sobre esto último, se espera que a mediano plazo se pueda contar con las herramientas necesarias para que las audiencias puedan ser grabadas en audio y en video siendo esta la forma ideal de verificar que efectivamente se cumplieron todas las garantías procesales.

Asimismo, la intervención de las TIC alcanzó a otras diligencias tales como inspecciones judiciales en las que la actuación es grabada en video, ${ }^{22}$ la producción de la prueba en la que se garantiza la equivalencia funcional, ${ }^{23}$ los remates judiciales que se

20. Ecuador, Código Orgánico General de Procesos, art. 4.

21. Ecuador, Código Orgánico Integral Penal, Registro Oficial 180, Suplemento, 10 de febrero de 2014, art. 565 .

22. Ecuador, Código Orgánico General de Procesos, art. 230.

23. Es decir, se le atribuye el mismo valor probatorio a los mensajes de datos y firmas electrónicas que en relación con los instrumentos escritos. Ecuador, Ley de Comercio Electrónico, Firmas Electrónicas y Mensajes de Datos, art. 14. 
realizan en línea, ${ }^{24}$ también se puede anticipar la consulta de expedientes que no han sido digitalizados y enviar solicitudes previas de copias simples y certificadas. Igualmente, con las últimas reformas, ya se trabaja en la implementación de plataformas electrónicas seguras para que los notarios tengan la posibilidad de realizar de forma telemática actos notariales a su cargo.

Ahora bien, todas estas actuaciones electrónicas, para que sean válidas y surtan los mismos efectos en términos de cumplimiento y ejecución, deben contar con firma electrónica. En relación con esto, la Ley Orgánica Reformatoria del Código Orgánico de la Función Judicial, ${ }^{25}$ dispuso que el Consejo de la Judicatura diseñe los sistemas electrónicos y virtuales adecuados y entregue las firmas y los certificados electróni$\cos ^{26}$ a los servidores públicos de la Función Judicial con el fin de facilitar la interconexión entre instituciones, priorizando aquellas competentes en materia de Familia. Este y otros aspectos normativos relevantes del tema en cuestión se encuentran regulados en la Ley de Comercio Electrónico, Firmas y Mensajes de Datos.

En cuanto a las notificaciones electrónicas, estas también están permitidas, y son válidas. ${ }^{27}$ En relación con la citación electrónica, los cambios hechos últimamente ya permiten realizar la citación por medios electrónicos. La Ley Orgánica Reformatoria del Código Orgánico de la Función Judicial manda la creación del Sistema Único de Notificación y Gestión de Trámites del Estado ${ }^{28}$ incluyendo el buzón electrónico ciudadano, ${ }^{29}$ en el que se deberá consignar un correo electrónico, autorizando notificaciones, de modo que para el 2024 toda persona mayor de edad y menor a 65 años deberá crear y usar de forma obligatoria el buzón ciudadano. ${ }^{30}$ En este sentido, el COGEP permite realizar la citación telemática en el buzón mediante boletas, previa notificación por la prensa, cuando no sea posible encontrar al demandando personalmente o identificar su domicilio, igualmente se tendrán presentes para esta citación los correos que hayan sido aceptados mediante un contrato. En el caso de personas jurídicas se podrá usar también el correo que conste registrado en el ente de control, mientras que a todos los órganos y entidades del sector público se les citará en forma telemática por el Sistema de Notificaciones Electrónicas. Reformas todas con las que

24. Ecuador Función Judicial, "Remates judiciales en línea", Función Judicial, accedido 10 de noviembre, https://bit.ly/3nPv2dS.

25. Ecuador, Ley Orgánica Reformatoria del Código Orgánico de la Función Judicial, Registro Oficial 345, Suplemento, 8 de diciembre de 2020.

26. Definición en Ecuador, Ley de Comercio Electrónico, Firmas Electrónicas y Mensajes de Datos, art. 20.

27. Ecuador, Código Orgánico General de Procesos, art. 66.

28. Ecuador, Ley de Comercio Electrónico, Firmas Electrónicas y Mensajes de Datos, art. 12.1.

29. Ibíd., art. 12.2.

30. En España mediante la Sede Judicial Electrónica. España, Ley 18/2011, art. 9. 
se pretende agilizar el proceso de citación que genera demora y dilata la gran mayoría de los trámites, a la espera de que con la implementación efectiva de esta modificación medular, no se hagan tambalear principios y garantías procesales.

De esta forma inicia en Ecuador la justicia electrónica, de a poco se presentan peticiones y documentos digitales, ya estamos más pendientes de nuestra casilla judicial electrónica que de una física, ya preferimos audiencias remotas; así es como Ecuador apuesta por la inclusión de los avances tecnológicos en aras de alcanzar una justicia que funcione al día, confiados en que por estos medios se den nuevas soluciones para viejos problemas.

\section{TOMA DE DECISIONES}

En este punto, vamos a dejar sentados los avances que hay en otros países puesto que en Ecuador no se han incluido las TIC como apoyo directo para la toma de decisiones, hoy en día los jueces deciden valorando individualmente la prueba, de acuerdo a las reglas de la sana crítica, no tienen a su disposición ningún tipo de software —a más de los indicados - que sirva de apoyo y guíe su decisión, tampoco existen sentencias o resoluciones que se hayan emitido consultando un asistente judicial virtual o sistemas similares que hagan uso de técnicas de inteligencia artificial, esta práctica en Ecuador no es convencional y aún genera resistencia.

Contrario a lo que sucede en Ecuador, el mundo trabaja arduamente por desarrollar sistemas computacionales que sirvan de apoyo a las decisiones judiciales, poniendo una vez más la tecnología al servicio del derecho y de la justicia. Ya son reales los sistemas de asesoramiento legal, programas que interpretan los datos que han sido ingresados, analizan normas y precedentes y devuelven una explicación concreta y clara del asunto, ofreciendo como resultado una posible resolución en caso de que el conflicto llegue a una instancia judicial, es un tipo de sistema de predictibilidad; así, un usuario puede decidir si en función al posible resultado es más conveniente una forma alternativa de solución del conflicto, acceder a la justicia ordinaria o incluso mantenerse al margen de un litigio. Uno de los objetivos principales de estos sistemas es descongestionar los juzgados, si ya como antecedente se tiene que la posibilidad de un resultado favorable es baja, así gana el Estado y también el recurrente porque no gasta recursos innecesariamente.

Hoy, es posible que este tipo de programas puedan asistir también al juez en la toma de decisiones pues son capaces de valorar prueba aportada por las partes, circunstancias relacionadas al caso, jurisprudencia y doctrina para así sugerir una posible resolución, la inteligencia artificial incorporada a estos programas permite que sean 
capaces de considerar una gran cantidad de información en segundos y ofrecer un resultado, trabajo que a un ser humano le llevaría días.

Existen también los sistemas Sentencing Guidelines ${ }^{31}$ logrados con técnicas de deep learning, estos programas informáticos no solo imitan el razonamiento humano de un experto y como apoyo a la toma de decisiones pretenden uniformidad en los fallos sino que van más allá, alcanzan un análisis más profundo del perfil del infractor y evalúan diferentes variables para determinar la posibilidad de que el infractor pueda reincidir en conductas delictivas en un futuro, sugiriendo la pena a imponerse con atenuantes o agravantes, consecuencia de haber considerado todos estos factores, como una especie de justicia preventiva.

Ante esto, es evidente que la pretendida seguridad social mediante prevención se contrapone a los principios de no discriminación, de presunción de inocencia, del derecho a la defensa, de seguridad jurídica y otros tantos, porque hemos de preguntarnos ¿cuáles son los factores que favorecen a que un ser humano pueda delinquir en un futuro? ¿tal vez lo sea pertenecer a cierto estatus social? o ¿tal vez una nacionalidad?, o ¿un género en particular? En todo caso, para garantizar una coexistencia pacífica, hemos de enseñar a la máquina que existen unos límites para la toma de decisiones en función de determinadas reglas que restringen las opciones que pueden sugerir como sentencia para no violentar derechos y principios fundamentales. Las Sentencing Guidelines tienen aceptación en gran parte de los Estados Unidos, mientras que sistemas de apoyo a la toma de decisiones como Split-up, Expertius, SIES se han desarrollado y están vigentes en países como Australia y México.

Cuestión diferente es el uso de la tecnología, ya no como un apoyo a los procesos judiciales, sino como suplente del juez. Este uso de las TIC representa la más avanzada manifestación de la 4.0 revolución industrial en el área del derecho. El llamado juez robot procura solucionar conflictos ofreciendo soluciones apropiadas para casos concretos. Hoy en día, el juez robot ya tiene participación en algunos sectores como en el de consumo por ejemplo, en Europa la plataforma ODR, ${ }^{32}$ ofrece servicios de negociación, mediación, arbitraje, etc. Este programa toma decisiones optimizando los recursos de ambas partes y se usa frecuentemente para resolver disputas por compras en línea (e-resolving to e-shopping disputes). La pregunta es ¿será que un juez robot puede reemplazar al juez humano en la solución de controversias en otras ramas del derecho?

31. P. ej. United States Sentencing Commission, accedido 5 de noviembre de 2020, https://bit.ly/34U9PIj.

32. Online Dispute Resolution europe (ODR), accedido 27 de noviembre de 2020, https://bit.ly/2KPNdS0. 


\section{DEL PLANTEAMIENTO A LA REALIDAD: LOS RETOS DE LA E-JUSTICIA}

Ahora bien, así como las ventajas de la e-justicia son numerosas, también lo son los inconvenientes y las interrogantes que surgen. Resultan preguntas como $i$ se lesionarán los derechos fundamentales y las garantías procesales con la incorporación de las TIC? ¿Cuál es el límite que se le debe poner a esta ola de cambios tecnológicos? o es que ¿se debe avanzar a cualquier precio? ¿en qué ramas del derecho se puede aplicar esta modernización? o incluso ¿estamos preparados para aprender la lección o al final vamos a retroceder?

Evidentemente, ninguna de estas cuestiones es fácil de contestar; y ni siquiera viene al caso batallar sobre si nuestra legislación ofrece las respuestas o si es clara y explícita ante los nuevos escenarios generados por la tecnología, porque la respuesta es simple: no es ni clara, ni uniforme, ni explícita. No podríamos afirmar que para encontrar respuestas certeras, menos aún definitivas, hemos de remitirnos a nuestra normativa, si ni siquiera países con normas flexibles que en cierta medida se adaptan a las nuevas situaciones han podido seguirle el paso al sin número de nuevos eventos consecuencia de esta revolución tecnológica, menos podrán países como Ecuador que tienen sistemas legales rígidos.

La mencionada brecha entre la tecnología y la legislación ha existido, existe y existirá, ya de este contexto se deriva un gran inconveniente, téngase en cuenta por puntualizar una situación, que el uso de estas herramientas requiere de forma incuestionable que alimentemos máquinas con una gran cantidad de datos personales. Surgen inquietudes porque estamos conscientes de que estas plataformas pueden ser manipuladas, y las usamos sin ni siquiera tener una ley de protección de datos personales. Preocupa la seguridad, preocupa que estos vacíos normativos puedan obstaculizar la digitalización de la justicia tal como se espera. Por esta y muchas otras necesidades se requiere al menos una adaptación de las normas que regulan la protección de datos personales, la administración de justicia y el proceso judicial.

Además, un reto importante es la asignación de recursos económicos suficientes para la adquisición de los equipos y software que permitan la realización de todas las diligencias electrónicas de forma segura. Es evidente que al menos en una primera etapa, como la que atraviesa Ecuador, el costo de la e-justicia es bastante alto, se requiere suficiente capacidad de los servidores para almacenar cantidades inmensas de información, plataformas web y móviles bien logradas, eficientes, seguras y amigables con el usuario, un equipo de soporte técnico constante, entre otros elementos. Por ahora, los recursos digitales que se han adquirido y asignado para este fin aún son escasos. 
Igualmente, no podemos dejar del lado el hecho de que las TIC deberían ser una espada para pelear la guerra de la pobreza, del analfabetismo, y sin embargo, no se puede negar que en Ecuador el acceso a la tecnología difiere entre regiones, entre grupos económicos, sociales e incluso culturales y entonces ¿cómo una persona puede conectarse a una audiencia virtual si ni siquiera tiene acceso a una computadora o a internet? ¿cómo se alcanzarán los objetivos de la e-justicia si la ciudadanía no tiene acceso a las herramientas digitales básicas o no sabe cómo usarlas? El reto es grande, porque esta brecha digital no solo problematizará, sino que impedirá el progreso. En tal virtud, necesariamente, ha de encontrarse la manera de garantizar el acceso a la justicia frente a la implementación de tecnologías en igualdad de condiciones.

Ahora bien, cuando lo nuevo reemplaza a lo viejo, se dan cambios sistemáticos profundos en la sociedad, y como bien recordaremos, en el acontecer de la humanidad han sucedido otras grandes transformaciones que por supuesto generaron inquietud, incertidumbre y por tanto resistencia al cambio, tal como sucede hoy en día. Sin lugar a duda, asimilar la amplitud, la profundidad y la velocidad ${ }^{33}$ de esta nueva revolución, no es tarea fácil; hacerlo implica perder lo conocido, salir de la zona de confort y desarrollar nuevas destrezas y habilidades que permitan la adecuada adaptación al mundo tecnológico. Hay que trabajar en una nueva cultura, porque la adquisición de herramientas tecnologícas no basta por si sola, para alcanzar el cambio que se desea.

Ante esto, hay que aclarar que no solo funcionarios de la justicia están llamados a ser capacitados y a manejar estas herramientas a la perfección promoviendo el uso de los medios digitales en los juzgados como actores principales de esta transformación, sino sobre todo los abogados en libre ejercicio profesional que acudirán a la justicia, pues si no se logra una generalización del uso de estas herramientas entre ellos, si no aprenden a utilizarlas o si simplemente prefieren no usar los servicios digitales que se ponen a su disposición, entonces de poco o de nada servirá todo este esfuerzo, las cortes seguirán congestionadas, los usuarios seguirán insatisfechos, y en definitiva, no se habrán dado soluciones sino que incluso se habrán creado más diferencias como una suerte de desigualdad social creciente.

Otros debates fuertes con respecto a las audiencias virtuales y a la citación electrónica van surgiendo, es posible que se experimenten paros en el avance de la digitalización de los procesos judiciales por enfrentamientos entre los precursores de la e-justicia y expertos en la materia, dada la colisión entre la implementación de las TIC y los derechos fundamentales y garantías procesales, lo cual devendrá en un fuerte

33. Klaus Schwab, La Cuarta Revolución Industrial (Bogotá: El Tiempo Casa Editorial, 2016), 9. 
debate de si un proceso virtual va en contra de lo dispuesto en el art. 75 de la Constitución ecuatoriana.

\section{A MODO DE CONCLUSIÓN}

El mundo tal y como lo conocíamos ya no existe, las TIC han sido las responsables, y el sistema judicial no podía ser ajeno a estos cambios, tenía que adaptarse a las circunstancias del entorno para garantizar el acceso a la justicia de una manera ágil, efectiva y más transparente.

Este proceso en Ecuador tuvo un impulso importante a partir de la pandemia por COVID-19, y aunque es reciente y aún no se ha materializado, este empuje fue bastante útil para vencer la resistencia al cambio que ralentizaba la implementación de las herramientas tecnológicas en la administración de justicia. En este entorno, se hicieron evidentes la utilidad y las ventajas de la inclusión de las TIC y sin lugar a duda, hoy en día la e-justicia se presenta como una innovación radical en el área del derecho.

No obstante, hay que decir que no estábamos preparados para afrontar un cambio tan profundo, es por ello por lo que, lo recomendable será avanzar con una introducción escalonada en el ordenamiento jurídico de los posibles instrumentos disponibles aplicables al mundo judicial, habrá que ir empapando poco a poco los diferentes sectores, pero sin perder el norte y sin dejarse ofuscar por el adelanto tecnológico. Se debe tener especial precaución en la introducción de videoconferencias para audiencias, pues estas requieren que se respeten una serie de formalidades para garantizar su validez, la seguridad jurídica, la no afección de derechos fundamentales y una tutela judicial efectiva, coherente con un Estado de Derecho.

Sabemos de la necesidad de revisar las normas existentes e incorporar nuevas, es claro también que no podemos avanzar a cualquier precio aun cuando a simple vista las ventajas sobrepasen por mucho a las dificultades, hay que revisar una y otra vez que cada paso hacia delante vaya a ser definitivo, que garantice los derechos de las personas para no tener que retroceder.

Por ahora, construir el puente hacia la e-justicia sigue siendo un gran reto, se buscan soluciones a los problemas que se presentan en el camino para lograr la tan anhelada "Justicia Justa", somos conscientes de que la inclusión de cualquier avance proveniente de la 4.0 revolución industrial dentro de la justicia debe contar con una conditio sine qua non: jamás los adelantos de la tecnología en la justicia pueden hacerse a costa de los derechos y las libertades de los ciudadanos.

Finalmente, como país reconocemos que la tecnología no es el futuro sino que es el presente, que avanzamos hacia el día en el que las máquinas serán capaces incluso de crear su propio pensamiento, en el que las técnicas de inteligencia artificial y auto- 
matización se convertirán en pieza clave para la dinamización y modernización de la actividad judicial en el país, estamos conscientes de que en todo este proceso juega un papel primordial la ética, la protección de datos y principios procesales contenidos en el ordenamiento jurídico, sabemos que las nuevas tecnologías contribuyen a mejorar la calidad del servicio y la gestión interna de la administración de justicia. Asimismo, también percibimos las dificultades que se presentan, principalmente la falta de regulación en este campo, y los cuestionamientos que plantea su uso por una posible colisión de derechos, los debates éticos y morales que se han generado.

Y a pesar de ello, hemos de concluir diciendo que hemos asumido el reto, admitimos la evolución y la innovación tecnológica, y la aceptamos desde una visión positiva para aprovechar las potentes olas de las tecnologías exponenciales que traerán gigantescas oportunidades de crecimiento, veremos si logramos salir airosos de este proceso o si tantas olas terminan por hacer del día de playa muy poco atractivo.

\section{BIBLIOGRAFÍA}

Álvarez, Leonardo. "Justicia electrónica". Revista digital de Derecho Administrativo 4 (2010): 43-56. https://bit.ly/2KQyF4E.

Barona, Silvia. "Cuarta revolución industrial (4.0) o ciber industria en el proceso penal: revolución digital, inteligencia (2010): 1-21. doi:http://dx.doi.org/10.24822/rjduandes.0301.1.

—. "Inteligencia artificial o la algoritmización de la vida y de la justicia. ¿Solución o problema?". Revista Boliviana de Derecho 28 (2019): 18-49. https://bit.ly/381So46.

Batista Hernández, Noel. "La toma de decisiones en la informática jurídica basado en el uso de los sistemas expertos”. Revista Investigación Operacional 40, n. ${ }^{\circ} 1$ (2019): 131-9. https:// bit.ly/3pyrJbo.

Bueno de Mata, Federico. "E-justicia: Hacia una nueva forma de entender la justicia". Revista Internacional de Estudios de Derecho Procesal y Arbitraje 1 (2010): 1-10. https://bit. ly/38rOoiE.

Cáceres, Enrique. "Inteligencia artificial, derecho y e-justice (el proyecto iij-conacyt)". Boletín Mexicano de Derecho Comparado 116 (2006): 593-611. https://bit.ly/3nJEGyk.

Delgado, Ana María, y Rafael Cuello. "Iniciativas recientes de la e-justicia en España". Revista de Internet, Derecho y Política 4 (2007): 22-30. https://bit.ly/38wGqoy.

Ecuador. Consejo de la Judicatura. Resolución $n .^{\circ}$ 039-2020. Registro Oficial 217, 4 de junio de 2020.

-. Constitución de la República del Ecuador. Registro Oficial 449, Suplemento, 20 de octubre de 2008.

-. Código Orgánico de la Función Judicial. Registro Oficial 544, Suplemento, 9 de marzo de 2009. 
—. Código Orgánico General de Procesos. Registro Oficial 506, Suplemento, 22 de mayo de 2015.

—. Código Orgánico Integral Penal. Registro Oficial 180, Suplemento, 10 de febrero de 2014.

—. Función Judicial. "Sistema de Jurisprudencia". Función Judicial. Accedido 20 de diciembre. https://bit.ly/3qOoYmv.

-. Ley de Comercio Electrónico, Firmas Electrónicas y Mensajes de Datos. Registro Oficial 557, Suplemento, 17 de abril de 2002.

- Ley Orgánica Reformatoria del Código Orgánico de la Función Judicial. Registro Oficial 345, Suplemento, 8 de diciembre de 2020.

-. Proyecto de Ley Orgánica de Datos Personales. Memorando n. ${ }^{\circ}$ PAN-CLC-2019-0184, 19 de septiembre de 2019.

Estonia e-estonia. "E-justicia". Enterprise Estonia. Accedido el 26 de febrero. https://bit. ly/3uJvKNw.

España. Ley 18/2011 Reguladora del uso de las Tecnologías de la Información y la Comunicación en la Administración de Justicia. Boletín Oficial 160, 5 de julio de 2011.

Fabra, Pere. "E-justicia". Revista de Internet, Derecho y Política 4 (2007): 1-12. doi:http:// dx.doi.org/10.7238/issn.1699-8154.

García Barrera, Myrna. "Justicia electrónica: en busca de la interoperabilidad”. Instituto de Investigaciones Jurídicas de la UNAM. 2016. https://bit.ly/3azICya.

—. "Juzgado sin papel, un paso más de la justicia electrónica". Revista del Instituto de Ciencias Jurídicas de Puebla 41 (2017): 133-54. https://bit.ly/3mGknjW.

Gamboa, Rafael. "Validez procesal de la información digital". Revista de Derecho, Comunicaciones y Nuevas Tecnologías 1 (2005): 53-73. https://bit.ly/3nGzOKu.

González, Conrado, y Álvaro Gimeno. "La era de la justicia digital y las nuevas tecnologías en la administración de justicia”. Revista Acta Judicial 1 (2017): 72-88. https://bit. ly/37F07Lz.

González, Manuel. "La justicia electrónica en españa: qué es, cómo se regula y cómo funciona”. Revista de la Facultad de Derecho de México 269 (2017): 1032-74. doi:http://dx.doi. org/10.22201/fder.24488933e.2017.269.62495.

Hernández, Noel, et al. "La toma de decisiones en la informática jurídica basado en el uso de los sistemas expertos”. Revista Investigación Operacional 1 (2019): 131-9. https://bit. ly/3mGksnK.

Kevin, Ashley. "Designing Electronic Casebooks That Talk Back: The CATO Program”. Jurimetrics, vol. 40, n. 3 (Primavera de 2000): 275-319.

Inter-American Development Bank. "Digital Technologies for Better Justice". Inter-American Development Bank. 2020. https://bit.ly/34SznFu.

Lobos, Ricardo. "El Uso de Nuevas Tecnologías en el Sistema Judicial: experiencias y precauciones". Seminario de Gestión Judicial 1 (2010): 117-40. https://bit.ly/3rcY0Gq. 
Martínez Bahena, Goretty Carolina. "La inteligencia artificial y su aplicación al campo del Derecho". Alegatos 82 (2013): 827-46. https://bit.ly/3aKO411.

Micó, Josep-Lluís. “(In)justicia digital”. Trípodos 19 (2006): 113-28. https://bit.ly/3pffOPC.

Online Dispute Resolution europe (ODR). Accedido 27 de noviembre de 2020. https://bit. ly/2KPNdS0.

Ontanu, Elena Alina. “Adpating Justice to Technology and Technology to Justice: A Coevolution Process to e-Justice in Corss-border". European Quarterly of Political Attitudes and Mentalities 8, n. ${ }^{\circ} 2$ (2019): 54-74. https://bit.ly/3pfUXeY.

Reiling, Dory. "Beyond court digitalization with ODR". International Journal for Court Administration 8, n. ${ }^{\circ} 2$ (2017): 2-6. doi:http://doi.org/10.18352/ijca.225.

—. "Doing justice with information technology". Information \& Communicationes Technology Law 15 (2006): 189-200. doi:https://doi.org/10.1080/13600830600676685.

- "E-justice: experiences with court IT in Europe". Buenas prácticas para la implementación de soluciones tecnológicas en la administración de justicia 1 (2011): 79-115. https:// bit.ly/2Juu1ZJ.

-. Technology for justice: How Information Technology can support Judicial Reform. Leiden: Leiden University Press, 2009.

Rueda, Andrea, y Jeimy Cano. "Valoración de la evidencia digital: Análisis y propuesta en el contexto de la administración de justicia en Colombia". Revista de Derecho, Comunicaciones y Nuevas Tecnologías 2 (2006): 95-122. https://bit.ly/2JeQTfE.

Schwab, Klaus. La Cuarta Revolución Industrial. Bogotá: El Tiempo Casa Editorial, 2016.

Turbo Court. "Prepare your court case online". Accedido 17 de noviembre de 2020. https:// bit.ly/3rq6NoO.

United States Sentencing Commission. Accedido 5 de noviembre de 2020. https://bit. ly/34U9PIj.

Velicogna, Marco. "Electronic Access to Justice: From Theory to Practice and Back". Droit et Cultures 61 (2011). https://bit.ly/37WwKV8.

—. "In Search of Smartness: The EU e-Justice Challenge". Informa Tics 4 (2017): 1-17. doi:https://doi.org/10.3390/informa Tics4040038.

—. "Justice systems and ICT: What can be learned from Europe?". Utrecht Law Review 3 (2007): 129-47. doi:http://doi.org/10.18352/ulr.41.

Velicogna, Marco, Antonie Errera y Stéphane Derlange. "Building e-Justice in Continental Europe: The TéléRecours Experience in France". Utrecht Law Review 9 (2013): 38-59. doi:https://doi.org/10.18352/ulr.211. 